\title{
Introdução de Ligações Cruzadas no LLDPE Através de Processo de Extrusão Reativa de Graftização do Vinil-TriMetóxi-Silano (VTMS) na Cadeia Polimérica: Efeito das Condições de Processamento e do Sistema Reacional
}

\author{
Alexandre B. Pessanha, Marisa C. G. Rocha \\ Instituto Politécnico, UERJ \\ Antonio H. M. F. T. da Silva \\ Centro de Pesquisa Leopoldo Miguez, Petrobras
}

\begin{abstract}
Resumo: Embora a reticulação do polietileno efetuada através de processo de extrusão reativa, envolvendo a graftização de silanos vinílicos na cadeia polimérica, seja um processo bastante utilizado industrialmente, poucos estudos têm sido publicados sobre a influência das variáveis de processamento nas propriedades do polímero. Neste trabalho, foi avaliado o efeito das condições de processamento e do sistema reacional no índice de fluidez (MFI), no teor de gel e nas propriedades térmicas do polietileno linear de baixa densidade (LLDPE), graftizado com vinil-trimetóxi-silano (VTMS) na presença de baixas concentrações de peróxido de dicumila (DCP) e reticulado com água. Uma metodologia estatística demonstrou que o teor de silano é a variável de processamento mais significativa, quando a reação é efetuada utilizando $0,02 \%$ p/p de DCP. Aumento significativo do teor de gel do LLDPE foi obtido utilizando uma concentração maior de DCP $(0,07 \%$ p/p). O processo de formação de ligações cruzadas causou aumento na estabilidade térmica e redução significativa no grau de cristalinidade, nas temperaturas de fusão e de cristalização e nas entalpias de fusão do LLDPE. Os espectros de infravermelho (FTIR) e o aumento da estabilidade térmica demonstram que houve formação de ligações cruzadas no polímero via pontes siloxano.
\end{abstract}

Palavras-chave: Polietileno, reticulação, peróxidos, silanos, graftização, extrusão.

\section{Silane Grafting and Mositure Crosslinking of LLDPE by a Reactive Extrusion Process: Effect of Processing Conditions and Reaction System}

\begin{abstract}
Although silane-modified polyolefins have become an industrial process, little data have been published on the dependence of silane grafting reactions and the properties of crosslinked polyolefins on reaction parameters. Here we studied the melt grafting of vinyl trimetoxi silane (VTMS) onto linear low density polyethylene (LLDPE) promoted by dicumyl peroxide (DCP) in a single screw extruder followed by crosslinking reaction in hot water. The influence of grafting formulations and processing conditions on the melt flow index (MFI), gel content and thermal properties of grafted and crosslinked LLDPE was evaluated using a statistical design. The silane content was the most important variable when the reaction was performed with a low concentration of DCP $(0.02 \mathrm{wt}$. (\%)). Samples with significant gel content, however, were obtained with 0.07 wt. (\%) of DCP. A decrease in the melt temperature and in the degree of crystallinity of LLDPE, as determined by differential scanning calorimetry (DSC) melting enthalpies, was observed as a result of crosslinking formation. The thermogravimetric analysis (TGA) showed an improvement in the thermal stability of LLDPE due to the introduction of crosslinking reactions. Results from Fourier Transformed Infrared Spectroscopy (FTIR) provided evidence for VTMS grafting onto LLDPE and subsequent crosslinking reaction.
\end{abstract}

Keywords: Polyethylene, crosslinking, peroxides, silanes, grafting reaction, extrusion.

\section{Introdução}

A modificação química do polietileno, através de processos de formação de ligações cruzadas, possibilita a sua utilização em aplicações que demandem elevadas temperaturas, assim como dá origem a um material com propriedades físico-mecânicas superiores. Comparado com o polímero não modificado, o polietileno reticulado apresenta maior resistência ao impacto, maior resistência química, melhor estabilidade dimensional, boa resistência à abrasão, propriedades elétricas superiores e maior resistência à propagação de trincas e à deformação $0^{[1-9]}$.

Atualmente, os principais métodos empregados industrialmente para a reticulação de polietileno são: utilização de peróxidos ou silanos como agentes de modificação química (iniciação térmica), ou uso de radiação com ou sem a adição de peróxidos (iniciação por radiação) ${ }^{[2,5]}$.
A técnica de reticulação de polietileno através da graftização com silanos vinílicos promovida por um peróxido adequado tem sido bastante estudada nos últimos anos em função das várias vantagens que oferece, como: razão custo-benefício razoável; a não necessidade de equipamentos caros; e a possibilidade de obtenção de uma estrutura tridimensional com propriedades mecânicas relevantes, embora apresente baixa densidade de ligações cruzadas. Este processo caracteriza-se pela graftização de um silano vinílico no polietileno, promovida pela ação de um peróxido orgânico adequado, seguida por hidrólise e condensação dos grupos silanol gerados ${ }^{[3]}$ (Figura 1). Esta reação é otimizada através da adição de catalisadores organometálicos ${ }^{[10-12]}$.

Industrialmente, os processos de reticulação do polietileno através de silanos são efetuados em extrusora em uma única etapa 


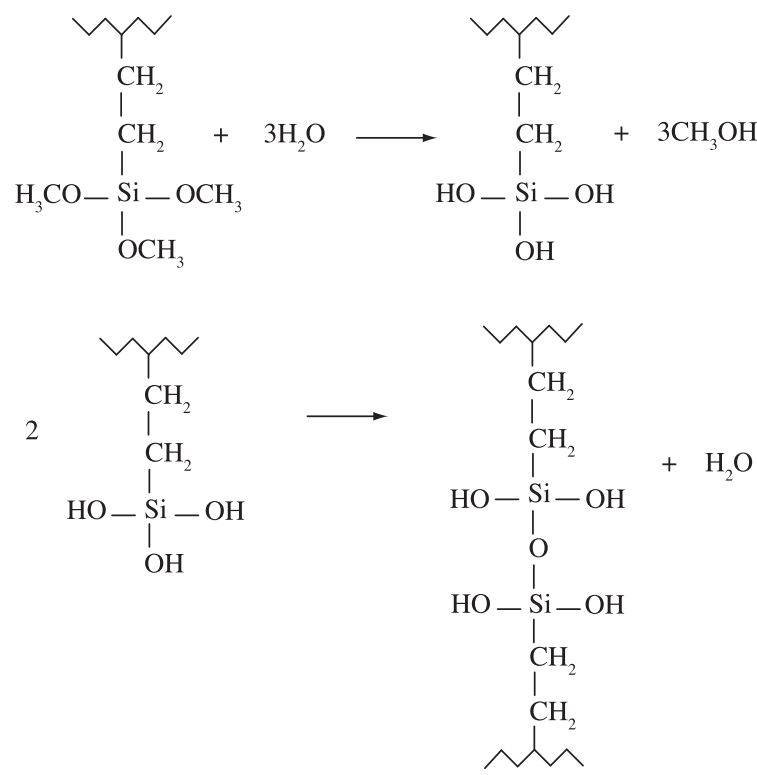

Figura 1. Formação de ligações cruzadas através de formação de pontes siloxano.

(Monosil) ou em duas etapas (Sioplas) ${ }^{[1,3,4,13]}$. Estes processos, entretanto, devem ser ajustados em função da extrusora utilizada, da configuração do parafuso, do protocolo de adição dos reagentes, dos parâmetros operacionais e das propriedades de desempenho desejadas. Poucos dados, entretanto, têm sido publicados sobre a dependência da reação de graftização e das propriedades do polímero reticulado das condições reacionais e de processamento ${ }^{[12,14,15]}$.

O polietileno linear de baixa densidade (LLDPE) por apresentar ramificações curtas aleatoriamente distribuídas na cadeia principal apresenta uma grande tendência às reações de extensão de cadeia. Em consequência deste fator e a quase contínua região amorfa que o torna mais suscetível à umidade, o LLDPE graftizado é reticulado sem requerer altas conversões ou tempos longos de reação ${ }^{[3,12,14,16]}$.

Há necessidade da adição de peróxido para que a graftização do silano ocorra. O peróxido de dicumila (DCP) é o iniciador mais utilizado nos processos de modificação do polietileno. Nos processos de reticulação do LLDPE, este peróxido é geralmente utilizado em concentrações muito baixas, o que é desejável para que a coloração do polímero seja mantida. Ademais, a utilização de baixas concentrações de peróxido em processos de modificação química de polietileno efetuados em extrusora tem sido apontada como um método efetivo de modificação das propriedades moleculares deste polímero e de suas propriedades ${ }^{[17]}$.

Nos métodos que envolvem a adição de silanos, a extensão da graftização parece ser regulada tanto pela concentração de silano quanto pela concentração de peróxido. Geralmente há um aumento do teor de gel com o aumento da concentração de peróxido e de silano até uma dada concentração a partir da qual não há alterações significativas das propriedades ${ }^{[3]}$.

O objetivo deste trabalho é estudar o processo de formação de ligações cruzadas no LLDPE, através da graftização de silanos vinílicos na cadeia polimérica, efetuada via processamento reativo, e avaliar o efeito das condições de processamento e do sistema reacional no índice de fluidez (MFI), no teor de gel e nas propriedades térmicas do LLDPE reagido com vinil-trimetóxi-silano (VTMS) na presença de baixas concentrações de DCP.

\section{Experimental}

Materiais

O polímero utilizado foi o polietileno linear de baixa densidade (LLDPE), código: RA-34U3, índice de fluidez de 4,2 g / $10 \mathrm{~min}$ $\left(190{ }^{\circ} \mathrm{C}, 2,16 \mathrm{~kg}\right.$ ), fornecido pela empresa Politeno Indústria e Comércio S.A. A resina, na forma de pó, apresenta em sua formulação 900 ppm de antioxidante e 2500 ppm de estabilizante à luz. As características e propriedades do LLDPE são apresentadas na Tabela 1. O peróxido de dicumila (DCP), Perkadox BC-FF, com tempo de meia vida $\left(\mathrm{t}_{1 / 2}\right)$ de 5,4 segundos $\left(200{ }^{\circ} \mathrm{C}\right)$ e temperatura de degradação igual a $177{ }^{\circ} \mathrm{C}$ foi fornecido pela Akzo Nobel Chemicals. O agente de graftização usado nesse estudo foi o vinil-trimetóxi-silano (VTMS), Z-6518, fornecido pela empresa Dow Corning do Brasil LTDA.

\section{Métodos \\ Graftização do VTMS na cadeia do LLDPE e formação de ligações cruzadas}

Inicialmente, o LLDPE foi submetido a um processo preliminar de secagem a $70{ }^{\circ} \mathrm{C}$, por um período de 24 horas, em uma estufa com circulação de ar. As pré-misturas constituídas por LLDPE, DCP $(0,02$ e $0,07 \%$ p/p) e VTMS $(0,5,1,0$ e $1,5 \%$ p/p) foram inicialmente processadas em um misturador WAB, modelo T2F, durante 10 minutos. Em seguida, as amostras foram processadas em extrusora monorosca (Wortex Wex 30, diâmetro $(\Phi)=30 \mathrm{~mm}$ ) em velocidades de rotação de parafuso de 10 e $50 \mathrm{rpm}$. O perfil de temperatura adotado nas fases da extrusora, da zona de alimentação até a dosagem foi de 150/190/200/200/200 ${ }^{\circ} \mathrm{C}$. De forma a promover a formação de ligações cruzadas no LLDPE, as amostras extrusadas na forma de fitas foram imersas em banho de água quente a $80^{\circ} \mathrm{C}$ por 24 horas.

\section{Determinação do índice de fluidez}

O índice de fluidez (MFI) do LLDPE e dos materiais processados foi determinado em plastômetro de extrusão (Tinus Olsen, Mod. MP993 a) padronizado de acordo com a Norma ASTM D-1238, a $190{ }^{\circ} \mathrm{C}$, com carga total aplicada de $2,16 \mathrm{~kg}^{[3]}$.

\section{Determinação do teor de gel}

O teor de gel das amostras foi determinado através da técnica com extração do solvente descrita na Norma ASTM D-2765 ${ }^{[3]}$. As amostras cortadas em pequenos pedaços foram pesadas e submetidas a processo de refluxo em xileno a $120{ }^{\circ} \mathrm{C}$ por 10 horas, em extrator Soxhlet. Após este período, as amostras foram secas até peso constante em estufa a vácuo, a $60{ }^{\circ} \mathrm{C}$. O teor de gel foi obtido através da seguinte relação:

$$
\text { Teor de Gel }=\frac{W s}{W i} \times 100
$$

onde:

- Ws = massa da amostra não solúvel em xileno (g); e

- $\mathrm{Wi}=$ massa inicial da amostra $(\mathrm{g})$.

\section{Determinação das propriedades térmicas}

As temperaturas de fusão (Tm), de cristalização (Tc) e as entalpias de fusão $(\Delta \mathrm{Hm})$ foram obtidas através da calorimetria exploratória diferencial (DSC-Q-100, TA Instruments). As análises foram efetuadas em atmosfera inerte de nitrogênio, utilizando taxas de aquecimento e de resfriamento de $10{ }^{\circ} \mathrm{C} / \mathrm{min}$. O grau de cristalinidade das amostras em porcentagem $(\mathrm{Xc} \%)$ foi determinado a partir das entalpias de fusão. $\mathrm{O}$ valor adotado para a entalpia de fusão para o polietileno $100 \%$ cristalino foi de $293 \mathrm{~J}^{-\mathrm{g}^{-1[3]}}$. 


\section{Avaliação da resistência à degradação térmica dos materiais}

A avaliação da resistência à degradação térmica dos materiais foi efetuada através de análise termogravimétrica (TGA-Q 50, TA Instruments). As análises foram efetuadas em atmosfera de nitrogênio, a uma taxa de aquecimento de $20^{\circ} \mathrm{C} / \mathrm{min}$

Determinação dos grupos funcionais dos produtos das reações de graftização e de formação de ligações cruzadas no LLDPE

A identificação de grupos funcionais no polímero decorrentes das reações de graftização e, ou da formação de ligações cruzadas foi realizada através de espectrometria no infravermelho com transformada de Fourier (FTIR), em equipamento Perkin Elmer (Perkin Elmer 1720x, Excalibur Series 3100, Varian) dotado de refletância total atenuada (ATR), cristal de diamante e operando na região de 4000 a $600 \mathrm{~cm}^{-1}$.

\section{Planejamento fatorial de experimentos}

A metodologia de planejamento fatorial de experimentos foi utilizada para avaliar o efeito das variáveis: velocidade de rotação do parafuso da extrusora, concentração de silano e imersão em água quente no MFI dos produtos da reação do LLDPE com $0,02 \%$ p/p de DCP e VTMS $(0,5,1,0$ e 1,5\% p/p). A imersão em água quente foi efetuada com o intuito de promover a formação de ligações cruzadas. Os níveis das variáveis independentes utilizadas no planejamento de

Tabela 1. Especificações do LLDPE.

\begin{tabular}{lcr}
\hline \multicolumn{1}{c}{ Especificações } & ASTM & Valores \\
\hline Densidade $\left(\mathrm{g} . \mathrm{cm}^{-3}\right)$ & $\mathrm{D}-792$ & $0,935^{\mathrm{a}}$ \\
Índice de fluidez $\left(2,16 \mathrm{Kg} / 190{ }^{\circ} \mathrm{C}\right)(\mathrm{g} / 10 \mathrm{~min})$ & $\mathrm{D}-1238$ & $4,2^{\mathrm{b}}$ \\
Temperatura de degradação $\left({ }^{\circ} \mathrm{C}\right)$ & - & $442^{\mathrm{b}}$ \\
Temperatura de fusão $\left({ }^{\circ} \mathrm{C}\right)$ & - & $122^{\mathrm{b}}$ \\
Temperatura de cristalização $\left({ }^{\circ} \mathrm{C}\right)$ & - & $111^{\mathrm{b}}$ \\
Resistência à quebra sob tensão ambiental $(\mathrm{h} / \mathrm{F} 50)$ & $\mathrm{D}-1693$ & $400^{\mathrm{a}}$ \\
Resistência à tração no escoamento $(\mathrm{MPa})$ & $\mathrm{D}-638$ & $17^{\mathrm{a}}$ \\
Alongamento na ruptura $(\%)$ & $\mathrm{D}-638$ & $22^{\mathrm{a}}$ \\
Módulo de flexão $(\mathrm{MPa})$ & $\mathrm{D}-747$ & $450^{\mathrm{a}}$ \\
Resistência ao impacto Izod $\left(\mathrm{J} . \mathrm{m}^{-1}\right)$ & $\mathrm{D}-256$ & $180^{\mathrm{a}}$ \\
Ponto de amolecimento Vicat $\left({ }^{\circ} \mathrm{C}\right)$ & $\mathrm{D}-1525$ & $116^{\mathrm{a}}$ \\
\hline
\end{tabular}

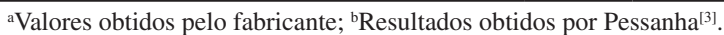

experimentos foram: teor de silano $(0,5 \% \mathrm{p} / \mathrm{p}, 1,5 \mathrm{p} / \mathrm{p})$; imersão das amostras em água quente: (sem imersão e com imersão); velocidade de rotação da extrusora (0 e $50 \mathrm{rpm})$. O tratamento estatístico dos dados foi efetuado utilizando o software Statistica.

\section{Resultados e Discussão}

O índice de fluidez é um parâmetro definido empiricamente, inversamente proporcional à viscosidade do material fundido a uma temperatura e taxa de cisalhamento, especificadas pelas condições operacionais de medida. Sendo assim é dependente de propriedades moleculares, tais como massa molar e ramificações, bem como da distribuição dessas propriedades. Ademais, é criticamente influenciado pelas condições de medida ${ }^{[3]}$. Devido à simplicidade da técnica empregada na determinação deste parâmetro, dados de MFI foram obtidos neste trabalho para acompanhar o processo de extensão de cadeia e formação de ligações cruzadas no polietileno. São apresentados na Tabela 2, os resultados da determinação do índice de fluidez (MFI) dos produtos de reação do LLDPE com DCP e silano, nas velocidades de extrusão de 10 e 50 rpm. É válido ressaltar que o resultado tabelado corresponde à média dos valores determinados.

Pode ser observado que a concentração fixa de $0,02 \% \mathrm{p} / \mathrm{p}$ de DCP, à medida que o teor de silano é aumentado no sistema reacional, há uma redução nos valores de MFI. Este resultado talvez possa ser atribuído às reações de extensão de cadeia que dão origem a moléculas com massa molar mais elevada, causadas pela ação do peróxido, uma vez que praticamente não houve alteração do MFI, após imersão das amostras em água, indicando que não houve formação de ligações cruzadas através de formação de pontes siloxano. Em concentração mais elevada de DCP, 0,07\% $\mathrm{p} / \mathrm{p}$, o MFI das amostras não foi determinado porque as amostras não escoaram nas condições padronizadas usadas nos ensaios. A análise da Tabela 2 mostra também que valores significativos de gel são obtidos em concentração maior de peróxido e em teores mais elevados de silano. Pode ser observado que há um comportamento associado entre a redução do MFI e o surgimento de gel.

Dispersões nos valores de teor de gel obtidos em estudos de reticulação de polietileno têm sido observadas, principalmente em casos nos quais as amostras não foram reticuladas sob pressão e nos processos de formação de ligações cruzadas envolvendo silanos ${ }^{[3]}$. Este fato, aliado à baixa concentração de gel, pode explicar os

Tabela 2. Teor de gel e índice de fluidez (MFI) dos produtos de reação do LLDPE com DCP e silano (VTMS).

\begin{tabular}{|c|c|c|c|c|}
\hline \multirow[t]{4}{*}{ Sistema reacional } & \multicolumn{4}{|c|}{ Velocidade da rosca (rpm) } \\
\hline & \multicolumn{4}{|c|}{10} \\
\hline & \multicolumn{2}{|c|}{ Antes da imersão } & \multicolumn{2}{|c|}{ Após a imersão } \\
\hline & MFI (g/ $10 \mathrm{~min})$ & Teor de gel $(\%)$ & MFI (g/ 10 min) & Teor de gel $(\%)$ \\
\hline LLDPE + 0,02\% DCP + 0,5\% VTMS & 1,6 & 0,1 & 1,6 & 0 \\
\hline LLDPE $+0,02 \%$ DCP $+1,0 \%$ VTMS & 1,1 & 1,3 & 1,1 & 0 \\
\hline LLDPE + 0,02\% DCP + 1,5\% VTMS & 0,9 & 0,6 & 0,9 & 0 \\
\hline $\mathrm{LLDPE}+0,07 \% \mathrm{DCP}+0,5 \% \mathrm{VTMS}$ & (a) & 0,7 & (a) & 4,5 \\
\hline LLDPE + 0,07\% DCP + 1,0\% VTMS & (a) & 0 & (a) & 60,2 \\
\hline \multirow[t]{2}{*}{ LLDPE + 0,07\% DCP + 1,5\% VTMS } & (a) & 0 & (a) & 74,8 \\
\hline & \multicolumn{4}{|c|}{50} \\
\hline $\mathrm{LLDPE}+0,02 \% \mathrm{DCP}+0,5 \% \mathrm{VTMS}$ & 1,6 & 0 & 1,6 & 0 \\
\hline $\mathrm{LLDPE}+0,02 \% \mathrm{DCP}+1,0 \% \mathrm{VTMS}$ & 1,1 & 0 & 1,1 & 2,3 \\
\hline LLDPE $+0,02 \% \mathrm{DCP}+1,5 \% \mathrm{VTMS}$ & 1,1 & 0 & 0,9 & 0 \\
\hline $\mathrm{LLDPE}+0,07 \% \mathrm{DCP}+0,5 \% \mathrm{VTMS}$ & (a) & 0 & (a) & 1,8 \\
\hline LLDPE + 0,07\% DCP + 1,0\% VTMS & (a) & 0 & (a) & 33,0 \\
\hline LLDPE + 0,07\% DCP + 1,5\% VTMS & (a) & 1,5 & (a) & 44,5 \\
\hline
\end{tabular}

${ }^{\mathrm{a} M a t e r i a l ~ n a ̃ o ~ e s c o o u ; ~ N A ~-~ N a ̃ o ~ s e ~ a p l i c a . ~}$ 
resultados anômalos observados, como os valores de teor de gel mais elevados do que os obtidos após o processo de imersão em água.

O efeito da variação da velocidade da rosca do parafuso, da concentração de silano e do procedimento de imersão em água no MFI dos produtos obtidos da reação do LLDPE com $0,02 \%$ de DCP e concentração variável de silano foi avaliado através de planejamento fatorial de experimentos. É importante ressaltar que só foi possível aplicar a metodologia estatística para as condições experimentais: teor de silano $(0,5 \% \mathrm{p} / \mathrm{p}, 1,5 \mathrm{p} / \mathrm{p})$; imersão das amostras em água quente: (sem imersão e com imersão); velocidade de rotação da extrusora (10 e $50 \mathrm{rpm}$ ). Em concentração mais alta de DCP não foi possível obter os dados de MFI, devido à alta viscosidade dos materiais. A dificuldade de obtenção dos valores de teor de gel também não permitiu que um número de replicatas significativo, do ponto de vista estatístico, fosse obtido.

A análise de variância (ANOVA) e os efeitos principais e de interação entre as variáveis no MFI são apresentados na Tabela 3.

A análise de significância dos fatores pode ser efetuada através da inspeção dos valores da média quadrática (Mean Square - MS) pela razão $\mathrm{F}$ ou, especialmente, através dos valores da distribuição $\mathrm{p}^{[3]}$. Neste trabalho utilizou-se a distribuição $\mathrm{p}$ para analisar a significância dos efeitos. Quanto mais significativo for o efeito do fator sobre a variável de resposta, (MFI, neste caso) maiores serão os valores de MS e menores serão os valores da distribuição p. Os fatores são considerados significativos com $95 \%$ de confiança, se os efeitos apresentarem valores de $\mathrm{p}<0,05^{[3]}$.

A análise da Tabela 3 mostra que o teor de silano é a variável que influencia de forma significativa o MFI. A análise da significância dos efeitos de interação entre o fator: teor de silano e os fatores: rotação de parafuso e imersão em água mostra que os fatores em questão não devem ser analisados separadamente (valores de $\mathrm{p}$ muito menores que 0,05 ), tendo em vista que o valor do nível em que se encontra um dos fatores irá impactar na influência que o outro fator tem no índice de fluidez.

A análise dos efeitos mostra que o efeito principal do teor de silano $(-0,63)$ foi significativamente superior a todos os outros efeitos. O sinal negativo indica que o aumento do teor de silano no meio reacional provoca uma redução nos valores de MFI. A imersão em água e a velocidade do parafuso não afetam o MFI de forma isolada, mas a ação combinada do aumento do teor de silano e do processo de imersão em água causa uma redução dos valores de MFI, menor do que a causada pelo aumento da concentração de silano no sistema reacional. Estes resultados demonstram que para haver reticulação do LLDPE, através de formação de pontes siloxano, promovidas pela ação de $0,02 \%$ de DCP deve-se aumentar o teor de silano na reação. É importante mencionar que em todos os casos, os erros associados aos efeitos apresentam-se, em geral, com uma ordem de grandeza inferior, o que demonstra a qualidade dos resultados experimentais obtidos. $\mathrm{O}$ valor de $\mathrm{R}^{2}$ ajustado, neste caso, é igual a 0,92 , demonstrando que modelos de regressão lineares podem ser utilizados para modelar os dados; entretanto, a proposição e validação de modelos fogem ao escopo principal e objetivos deste trabalho.

No planejamento de experimentos para que a análise de variância seja válida é importante que premissas como distribuição normal para a variável de resposta e aleatoriedade e independência para as amostras analisadas sejam consideradas, pois somente a distribuição normal pode assegurar a determinação de desvios ${ }^{[3]}$. O histograma de resíduos, não apresentado neste trabalho, mostrou que a condição de normalidade da distribuição de resíduos está sendo atendida. Adicionalmente, testes estatísticos (Lilliefors e ShapiroWilks) asseguraram a validade da utilização de testes paramétricos para a análise dos fatores analisados sobre o MFI.

A Tabela 3 mostra o efeito pronunciado do aumento da concentração de DCP sobre o MFI e sobre o teor de gel. De uma forma geral, nos processos de formação de ligações cruzadas no polietileno via extrusão reativa, o aumento do teor de peróxido ao sistema reacional até uma concentração limite, ocasiona um aumento no teor de gel, redução no MFI e aumento na pressão da matriz da extrusora. Concentrações de aproximadamente $1 \%$ parecem ser suficientes para se obter $90 \%$ de reticulação ${ }^{[3]}$. Como mencionado anteriormente, as dificuldades inerentes à determinação do teor de gel das amostras não possibilitaram a análise estatística dos dados.

A Tabela 3 mostra também que valores mais elevados de teor de gel são obtidos em velocidades de extrusão mais baixas, possivelmente devido ao maior tempo de residência dos materiais na extrusora.

As propriedades térmicas do LLDPE e dos produtos de reação do LLDPE e VTMS na presença de DCP são apresentadas na Tabela 4.

As propriedades térmicas dos produtos da reação do LLDPE com VTMS promovida por 0,02 de DCP demonstram que houve redução do grau de cristalinidade do polímero. Este fato pode ser atribuído a reações de extensão de cadeia e, ou uma diminuta concentração de

Tabela 3. Análise de variância e dos efeitos principais e de interação entre as variáveis: teor de silano, velocidade de rotação do parafuso e imersão de amostras em água no MFI.

\begin{tabular}{|c|c|c|c|c|c|c|c|}
\hline \multirow[t]{2}{*}{ Fatores } & \multicolumn{7}{|c|}{$\begin{array}{l}\text { Efeitos estimados - Variável de resposta: MFI (g / } 10 \text { min) } \\
\qquad R^{2}=0,92713\end{array}$} \\
\hline & SS & Df & MS & $\mathbf{F}$ & $\mathbf{p}$ & Efeitos & Erro \\
\hline Interações Principais & & & & & & 1,08 & 0,01118 \\
\hline Bloco & 0,108 & 1 & 0,108 & 21,6 & $1,1 \times 10^{-5}$ & $-0,06$ & 0,01291 \\
\hline $\mathrm{X} 1$ teor de silano $\mathrm{L}$ & 7,938 & 1 & 7,938 & 1587,6 & 0 & $-0,63$ & 0,015811 \\
\hline $\mathrm{X} 2$ velocidade da rosca & 0,00833 & 1 & 0,00833 & 1,67 & 0,19981 & 0,01667 & 0,01291 \\
\hline $\mathrm{X} 1 \mathrm{X} 2$ & 0,012 & 1 & 0,012 & 2,5 & 0,00076 & 0,025 & 0,015811 \\
\hline X1X3 & 0,0605 & 1 & 0,0605 & 12,1 & 0,12463 & $-0,055$ & 0,015811 \\
\hline $\mathrm{X} 2 \mathrm{X} 3$ & 0,012 & 1 & 0,012 & 2,4 & 0,00049 & $-0,02$ & 0,01291 \\
\hline Ajuste ("lack of fit") & 0,22833 & 15 & 0,01522 & 3,04 & & & \\
\hline Erro puro & 0,48 & 96 & 0,005 & & & & \\
\hline SS Total & 9,72 & 119 & & & & & \\
\hline
\end{tabular}

SS: Soma dos quadrados mínimos; Df: graus de liberdade; Ms: Média quadrática; F: razão F; p: probabilidade de significância; R: coeficiente de correlação. Fonte: Programa STATISTICA versão 7.0610. 
Tabela 4. Propriedades térmicas do LLDPE e dos produtos de reação do LLDPE e VTMS na presença de DCP.

\begin{tabular}{cccccc}
\hline $\begin{array}{c}\mathbf{D C P} \\
(\boldsymbol{\%} \mathbf{p} / \mathbf{p})\end{array}$ & $\begin{array}{c}\text { VTMS } \\
(\boldsymbol{\%} \mathbf{p} / \mathbf{p})\end{array}$ & $\begin{array}{c}\mathbf{T}_{\mathbf{m}} \\
\left({ }^{\circ} \mathbf{C}\right)\end{array}$ & $\begin{array}{c}\mathbf{T}_{\mathbf{c}} \\
\left({ }^{\circ} \mathbf{C}\right)\end{array}$ & $\begin{array}{c}\Delta \mathbf{H} \text { fusão } \\
\left(\mathbf{J . g}^{-1}\right)\end{array}$ & $\mathbf{X}_{\mathbf{c}} \%$ \\
\hline 0 & 0 & 122,4 & 110,5 & 154,5 & 52,7 \\
0,02 & 0,5 & 124,4 & 111,8 & 132,1 & 45,1 \\
0,02 & 1,0 & 123,7 & 112,3 & 131,3 & 44,8 \\
0,02 & 1,5 & 123,7 & 112,3 & 130,9 & 44,7 \\
0,07 & 0,5 & 110,1 & 98,2 & 100,2 & 34,2 \\
0,07 & 1,0 & 109,1 & 97,7 & 89,4 & 30,5 \\
0,07 & 1,5 & 108,1 & 98,1 & 88,3 & 30,1 \\
\hline
\end{tabular}

ligações cruzadas, uma vez que não houve formação de gel nestas condições reacionais, mas houve redução dos valores de MFI. A redução da entalpia de fusão indica que houve alterações na rede cristalina que também pode ser atribuída à introdução de defeitos. As temperaturas de fusão e de cristalização, entretanto, não foram alteradas

A análise dos produtos das reações promovidas com 0,07\% $\mathrm{p} / \mathrm{p}$ de DCP mostra que houve uma redução significativa das propriedades térmicas ( $\mathrm{Tm}, \mathrm{Tc}, \Delta \mathrm{Hm}$ e $\mathrm{Xc}$ ) do polímero. Este fato pode ser atribuído à introdução de defeitos que dificultam o processo de formação de dobras de cadeia, causando assim uma redução do tamanho dos cristais lamelares, densidade do polímero e das temperaturas de fusão e de cristalização ${ }^{[3,18,19]}$. A restrição à mobilidade das cadeias poliméricas devido à formação de ligações cruzadas tem sido apontada também como um dos fatores que causam a diminuição do grau de cristalinidade do polímero $^{[3]}$. Segundo trabalho publicado na literatura ${ }^{[15]}$, o processo de graftização de silanos vinílicos no LDPE origina esferulitos menores e mais imperfeitos do que os do polímero virgem.

Os dados obtidos através de análise termogravimétrica (TGA) do LLDPE e dos produtos de reação do polímero com VTMS (1,0 e $1,5 \%$ ) na presença de $0,07 \%$ de DCP, em velocidades de rotação de rosca de 10 e 50 rpm são apresentados na Tabela 5.

Pode ser verificado que há um aumento da estabilidade térmica do polímero devido à formação de ligações cruzadas, uma vez que a temperatura na qual a degradação começa a ocorrer é aumentada em torno de $13 \%$. A temperatura na qual a velocidade de degradação do polímero é máxima também é levemente aumentada. Este resultado era esperado uma vez que a introdução de ligações cruzadas provoca uma redução da mobilidade das cadeias poliméricas, aumentando a resistência térmica. $\mathrm{O}$ aumento do teor de silano e da rotação do parafuso, entretanto, não causou um efeito significativo na estabilidade térmica do polímero.

A espectroscopia de infravermelho (FTIR) foi utilizada neste estudo para indicar a ocorrência de graftização do VTMS na cadeia do polietileno, assim como de formação de ligações cruzadas. $\mathrm{O}$ espectro do LLDPE puro e reagido com $0,07 \%$ de DCP e 1,5\% de silano a 50 rpm (após o banho) é apresentado na Figura 2.

A Figura 2 mostra a presença de um pico em torno de $1080 \mathrm{~cm}^{-1}$, atribuído à ligação $\mathrm{Si}-\mathrm{O}-\mathrm{CH}_{3}$, que foi detectado tanto nas amostras que foram expostas à água, como nas que sofreram processos de formação de ligações cruzadas apresentando ligações $\mathrm{Si}-\mathrm{O}-\mathrm{Si}$ $\left(1100 \mathrm{~cm}^{-1}\right)$, fato corroborado pelo aumento de estabilidade térmica através de TGA, como nas que sofreram somente reações de graftização. Uma banda em torno de $1244 \mathrm{~cm}^{-1}$, correspondente ao grupamento $\mathrm{Si}-\mathrm{CH}_{2}$, também pôde ser observada. O pico em $1595 \mathrm{~cm}^{-1}$ corresponde a uma deformação angular simétrica no plano, da molécula de água. Picos em torno de 2848, 2916, 1498 e $719 \mathrm{~cm}^{-1}$ foram atribuídos à deformação axial simétrica e assimétrica de $\mathrm{CH}_{2}$ e à deformação angular fora do plano de $\mathrm{CH}_{2}{ }^{[3]}$.
Tabela 5. Dados obtidos através da Análise Termogravimétrica (TGA) do LLDPE e dos produtos de reação do LLDPE com VTMS promovida por DCP.

\begin{tabular}{|c|c|c|c|c|c|}
\hline \multirow{2}{*}{\multicolumn{2}{|c|}{$\begin{array}{c}\text { Sistema } \\
\text { reacional }\end{array}$}} & \multicolumn{4}{|c|}{ Velocidade da rosca $(\mathrm{rpm})$} \\
\hline & & \multicolumn{2}{|c|}{10} & \multicolumn{2}{|c|}{50} \\
\hline $\begin{array}{c}\text { DCP } \\
(\% \mathrm{p} / \mathrm{p})\end{array}$ & $\begin{array}{l}\text { VTMS } \\
(\% \mathrm{p} / \mathrm{p})\end{array}$ & $\begin{array}{l}\mathbf{T}_{\text {onset }} \\
\left({ }^{\circ} \mathbf{C}\right)\end{array}$ & $\begin{array}{c}\mathbf{T}_{\text {vel máx }} \\
\left({ }^{\circ} \mathbf{C}\right)\end{array}$ & $\begin{array}{l}\mathbf{T}_{\text {onset }} \\
\left({ }^{\circ} \mathbf{C}\right)\end{array}$ & $\begin{array}{c}\mathbf{T}_{\text {vel. máx }} \\
\left({ }^{\circ} \mathbf{C}\right)\end{array}$ \\
\hline 0 & 0 & ND & ND & 442,2 & 471,6 \\
\hline 0,07 & 1 & 455,7 & 477,4 & 455,4 & 478,1 \\
\hline 0,07 & 1,5 & 461,8 & 481,9 & 456,0 & 476,8 \\
\hline
\end{tabular}

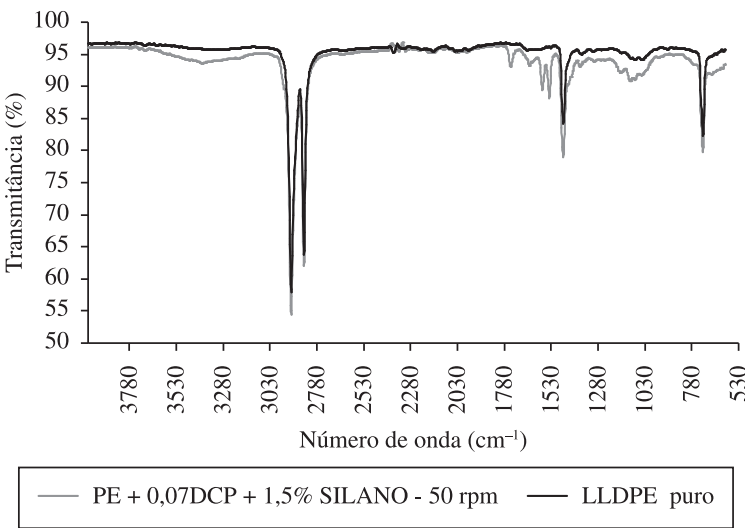

Figura 2. Espectro de infravermelho do LLDPE e do produto da reação do LLDPE com $1,5 \%$ p/p de VTMS promovida por $0,07 \%$ de DCP.

\section{Conclusões}

A concentração de silano é a variável de processamento que impacta de forma significativa os valores de MFI do LLDPE, quando a reação de formação de ligações cruzadas é efetuada utilizando $0,02 \%$ $\mathrm{p} / \mathrm{p}$ de DCP. O aumento da concentração de peróxido de $0,02 \% \mathrm{p} / \mathrm{p}$ para $0,07 \%$ p/p, cujo efeito no MFI não pôde ser avaliado através de planejamento de experimentos, causou um aumento significativo do teor de gel do LLDPE. A velocidade de extrusão mais baixa parece facilitar a formação de ligações cruzadas, possivelmente devido ao maior tempo de residência do polímero na extrusora. A reticulação do LLDPE influenciou de forma significativa a estabilidade térmica do polímero. O grau de cristalinidade e as temperaturas de fusão, de cristalização e as entalpias de fusão do LLDPE sofreram uma redução significativa devido à restrição na mobilidade das cadeias e à introdução de defeitos na região cristalina. Análise dos espectros de FTIR e os dados obtidos por TGA demonstram que houve formação de ligações cruzadas via pontes siloxano.

\section{Referências Bibliográficas}

1. Melo, R. P. - "Síntese e caracterização de polietileno reticulado para aplicação em dutos", Tese de Doutorado, Universidade Federal do Rio de Janeiro, Brasil (2009).

2. Kuan, H.-C.; Kuan, J.-F.; Ma, C.-C. \& Huang, J.-M. - J. Appl. Polym. Sci., 96, p.2383 (2005).

3. Pessanha, A. B. - "Modificação do LLDPE através de silanos viabilizando a aplicação em camadas plásticas de linhas flexíveis", Dissertação de Mestrado, Universidade do Estado do Rio de Janeiro, Brasil (2009).

4. Cameron, R.; Lien, K. \& Lorigan, P. - Wire J. Int., p.56 (1990).

5. Hjertberg, T.; Palmlöf, M. \& Sultan, B.-A. - J. Appl. Polym. Sci., 42, p.1185 (1991). 
6. Sirisinha, K.; Boonkongkaew, M. \& Kositchaiyong, S. - Polym. Test., 29, p.958 (2010).

7. Ahmed, G. S.; Gilbert, M; Mainprize, S. \& Rogerson, M. - Plas. Rubber and Compos., 38, p.13 (2009).

8. Azizi, H.; Morshedian, J. \& Barikani, M. - J. Vinyl Addit. Technol., p.184 (2009).

9. Barzin, J.; Azizi, H. \& Morshedian, J. - Polym. Plast. Technol., 45, p.979 (2006).

10. Sen, A. K.; Mukherjee, B.; Bhattacharyya, A. S.; De, P. P. \& Bhowmick, A. K. - Polym. Degrad. Stab., 39, p.281 (1992).

11. Konar, J.; Sen, A. K. \& Bhowmick - J. Appl. Polym. Sci., 48, p.1579 (1993).

12. Sen, A. K.; Mukherjee, B.; Bhattacharyya, A. S.; De, P. P. \& Bhowmick, A. K. - J. App. Polym. Sci., 44, p.1153 (1992).
13. Gross, L. H. - Wire Industry, p.609 (1990).

14. Lachtermacher, M. G. \& Rudin, A. - J. Appl. Polym. Sci., 59, p.1213 (1996).

15. Shieh, Y.-T. \& Tsai, T.-H. - J. Appl. Polym. Sci., 69, p.255 (1998).

16. Shieh, Y.-T. \& Liu, C.-M. - J. Appl. Polym. Sci., 74, p.3404 (1999).

17. Ramos, V. D.; Costa, H. M.; Pereira, A. O.; Rocha, M. C. G. \& Gomes, A. S. - Polym. Testing, 23, p.949 (2004).

18. Krupa, I. \& Luyt, A. S. - J. Appl. Polym. Sci., 81, p.973 (2001).

19. Kim, Y. C. \& Yang, K. S. - Polym. J., 31, p.579 (1999).

Enviado: 06/04/10

Aceito: $02 / 08 / 10$

DOI: $10.1590 / \mathrm{S} 0104-14282011005000018$ 